\title{
SOLITON-SOLITON INTERACTION IN CONFINING MODELS
}

\author{
Yu.A. SIMONOV ${ }^{1}$ \\ Physics Department, Free University of Amsterdam, Amsterdam, The Netherlands \\ and
}

\author{
J.A. TJON \\ Institute for Theoretical Physics, University of Utrecht, 3508 TA Utrecht, The Netherlands
}

Received 6 June 1979

\begin{abstract}
Solitons in confining models of the Werle type are considered in one spatial dimension and the interaction between them is studied numerically in detail. In the collision process new localized objects are generated, which are pulsating in time. Evidence is given about their stability.
\end{abstract}

Some classical field theoretical models have recently been studied by Werle [1] and Morris [2,3], which possess the remarkable property that all solutions have a non-dissipative character. Moreover, these models, which for brevity we call confining models, admit onesoliton-like solutions in four space-time dimensions. They might provide a good classical description of the bag idea [4]. In order that these solutions are physically acceptable they should be stable objects. Also the question of complete integrability of these types of models is of interest.

In this letter some aspects of these problems are studied. In particular, we present results for a confining model in one dimension, which show that the interaction between solitons can be strong and that this leads to the production of one or several objects with a qualitatively different structure than the one-soliton solution. Consider a lagrangian for a complex field,

$\mathcal{L}=\left|\partial_{t} \psi\right|^{2}-|\nabla \psi|^{2}-V\left(|\psi|^{2}\right)$,

in any space dimension with

$$
V\left(|\psi|^{2}\right)=\mu^{2}|\psi|^{2}+\lambda|\psi|^{2} b\left(|\psi|^{2}\right) .
$$

For confining models $b(y)$ satisfies for $y>0$ the property

\footnotetext{
1 Permanent address: ITEP, Moscow, USSR.
}

$b(y) \geqslant 0$,

$b(y) \rightarrow \infty, \quad y b(y) \rightarrow 0 \quad$ for $y \rightarrow 0$,

The asymptotic behaviour of the solutions of the Euler-Lagrange equations with the explicit time dependence

$\psi(r, t)=\mathrm{e}^{-\mathrm{i} \Omega t} \phi(r)$,

can simply be related for a certain class of $V$ to the type of non-linearity occurring in the interaction. For

$b(y)=|\ln y|^{\alpha /(1+\alpha / 2)}, \quad \alpha>0$,

we have $\phi \sim \exp \left(-c r^{1+\alpha / 2}\right)$, while for $b(y)=\ln ^{2} y$, $\phi \sim \exp (-c \cosh (2 \sqrt{\lambda r}))$. For even more singular $b(y)$ this solution decreases faster and finally in the case $b(y)=y^{-\alpha / 2}, 0<\alpha<1$ the one-soliton solution is exactly zero outside some radius. This last property was first discovered in ref. [1] for a specific model.

Since one of our main objectives is to study the interaction process between solitons and the situation with more than one spatial dimension is numerically more difficult to treat, we have looked for models which allow analytic solutions in one dimension. The two models we have considered are

$V=\mu^{2}|\psi|^{2}+\lambda|\psi|^{2-\alpha}, \quad \lambda>0, \quad 1>\alpha>0$,

and 
$V=\mu^{2}|\psi|^{2}+\nu^{2}|\psi|^{2} \ln ^{2}|\psi|^{2}$.

The stability of the soliton solutions of the form (4) for small perturbations can in principle be studied [5] by linearizing the equations of motion about this solution. To have stability it is sufficient that all eigenvalues of the resulting quadratic form should be nonnegative. A sufficient condition for this is

$\delta^{2} V\left(|\psi|^{2}\right) / \delta \psi \delta \psi^{*} \geqslant 0$

Since the charge

$Q=-\mathrm{i} \int\left(\psi \partial_{t} \psi^{*}-\psi^{*} \partial_{t} \psi\right) \mathrm{d} \boldsymbol{r}$

is conserved, the presence of one negative eigenvalue is still allowed as long as

$\mathrm{d}^{2} E(Q) / \mathrm{d} Q^{2}<0$

for the soliton solution [7]. As can be seen from energy and charge conservation the concavity condition (9) forbids the decay of one soliton into more solitons. The model (5) satisfies clearly the condition (7), while for sufficiently small $\nu$ this is also the case for (6).

So far our considerations are general and referred to a space of any dimension. In one space dimension the one-soliton solutions of the interactions (5) and (6) can explicitly be written down. In the c.m. system of a soliton they are given by

$$
\begin{aligned}
\phi_{\mathbf{S}}(x)= & \left(\lambda / \kappa^{2}\right)^{1 / \alpha}\left(\cos \frac{1}{2} \alpha \kappa x\right)^{2 / \alpha} \\
& \text { for }|x|<\pi / \alpha \kappa \equiv x_{0}, \\
= & 0 \text { for }|x|>x_{0},
\end{aligned}
$$

for the choice of (5) and

$\phi_{\mathrm{s}}(x)=\exp [-(\kappa / 2 v) \cosh (2 \nu x)]$,

for (6). In both cases we take $\mu^{2}>0$ and $\kappa^{2}=\Omega^{2}$ $-\mu^{2}>0$. The explicit solution of a moving soliton can simply be found from these by the application of a proper Lorentz transformation. For the solution (11) the linearized stability equation was studied and found to admit at most one negative eigenvalue. To show the stability of the solitons for the range of values of $\nu^{2}$ used in the numerical calculations $\left(\nu^{2} \leqslant 4\right)$ the dispersion curve $E(Q)$ was computed and indeed found to satisfy eq. (9).

For these one-dimensional models we have studied numerically the interaction process between solitons by letting two solitons collide on each other. The initial condition was chosen such that the two solitons are well separated in space and they approach each other with opposite velocity $u=u_{1}=-u_{2}$. Asymptotically for $t \rightarrow-\infty \psi$ has the form

$$
\begin{aligned}
\psi & =\exp \left[-\frac{\mathrm{i} \Omega_{1}}{\left(1-u^{2}\right)^{1 / 2}}(t-u x)\right] \phi_{\mathrm{s}}\left(\frac{x-u t-a_{1}}{\left(1-u^{2}\right)^{1 / 2}}\right) \\
& +\mathrm{e}^{\mathrm{i} \theta} \exp \left[-\frac{\mathrm{i} \Omega_{2}}{\left(1-u^{2}\right)^{1 / 2}}(t+u x)\right] \phi_{\mathrm{s}}\left(\frac{x+u t-a_{2}}{\left(1-u^{2}\right)^{1 / 2}}\right) .
\end{aligned}
$$

Note that for the model (5) eq. (12) is an exact solution to the equation of motion in view of eq. (10) as long as the solitons do not overlap. Several cases were considered in detail:

(1) $\Omega_{1}=\Omega_{2} \equiv \Omega, \theta=0$, symmetric soliton-soliton collision,

(2) $\Omega_{1}=\Omega_{2} \equiv \Omega, \theta=\pi$, anti-symmetric soliton-soliton collision,

(3) $\Omega_{1}=-\Omega_{2}=\Omega, \theta=0$, symmetric soliton-antisoliton collision.

In addition, some nonsymmetric situations have been studied corresponding to the scattering of solitons with different charges $\left(\left|\Omega_{1}\right| \neq\left|\Omega_{2}\right|\right)$. The calculations were done by putting the solitons on a ring which was 40 long. The $x$-values were discretized with 200 mesh points with mesh size $\Delta x=0.2$. The time integrations were done using the Numerov method and time steps of $\Delta t=0.002$. As a testing case the one-soliton expression with $u=0.5, \Omega=1.5$ was used as initial condition at $t=0$ and the time dependence was calculated up to $t=400$. Up to these times the numerical solution was in accordance with the one-soliton expression. Also in all the calculations of the time dependence of $\psi$ it was explicitly verified that the total energy and charge was conserved up to four decimals.

We now turn to discuss the results. Most of the calculations were done using the interaction (5) with $\alpha$ $=\frac{1}{2}, \mu=1$ and $u=0.5$. The values of the parameters $\lambda$ and $\Omega$ were taken in the range $0.5 \leqslant \lambda \leqslant 1.5$ and 1.4 $\leqslant \Omega \leqslant 1.7$. In all cases considered the collision was inelastic. For $\theta \neq \pi$ the solitons were passing each other, producing an additional lump of matter and coming out with less velocity than before the collision. A typical situation is shown in fig. 1 in the $x-t$ plane for $\lambda$ $=1$ and $\Omega=1.5$. As initial condition the two solitons were taken to be centered symmetrically around $x$ 


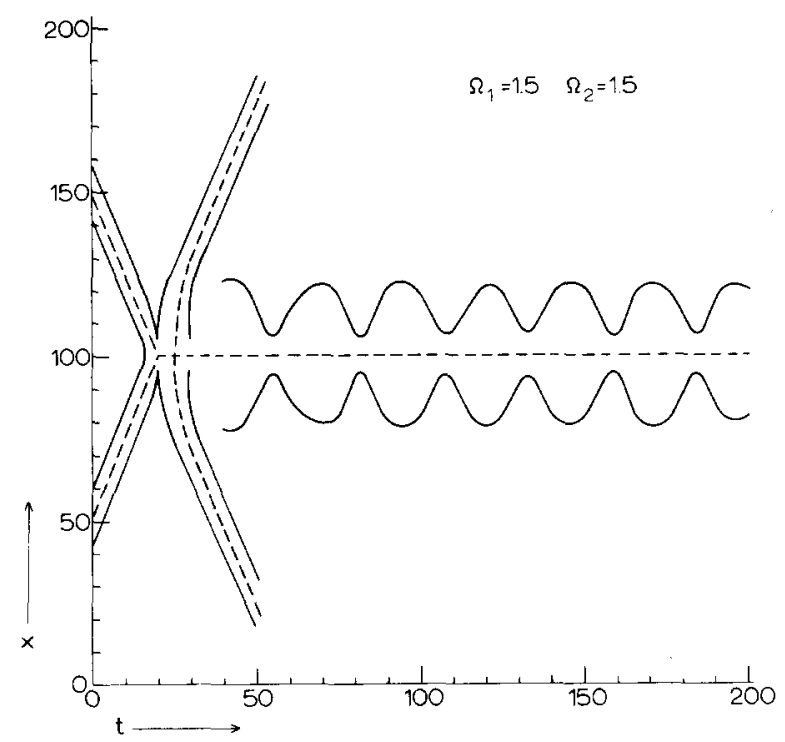

Fig. 1. The symmetric soliton-soliton collision with $\lambda=1$ displayed in the $x-t$ plane. The dashed lines represent the positions of the maxima of the solitons and the ss-breather, while the nearby solid lines mark the position where the amplitude has decreased half way.

$=100$ in units of $\Delta x=0.2$. Two important properties can be seen from this figure. The solitons suffer some delay in time during their interaction, typically of the order of several time units and the newly created object at around $x=100$ (to be called a ss-breather) is pulsating periodically and does not dissipate in space with time after the complete separation from the outgoing solitons. In fig. 2 are shown the magnitude $A$ and the time derivative $\mathrm{d} \varphi / \mathrm{d} t$ of the phase of the field at the center of the ss-breather as a function of $t$. Both vary periodically in time with $t_{\text {period }} \approx 26$. Furthermore when $A$ becomes maximal, d $\varphi / \mathrm{d} t$ is large. Also the shape dependence of $A$ in $x$ space changes in time as we can see from fig. 3. Here $A(x)$ and the energy density $\epsilon(x)$ are displayed at two moments. When $A(100)$ is maximal the shape is sharp while it broadens considerably at the times when $A(100)$ is small. Several studies of the ss-breather were done. Firstly we computed its charge $Q_{\mathrm{m}}$ and energy $E_{\mathrm{m}}$. These values lie in all cases considered slightly higher $(\sim 5 \%)$ than the one-soliton dispersion curve $E(Q)$. A remarkable property is that although the energy variation of the original solitons for the various initial conditions were

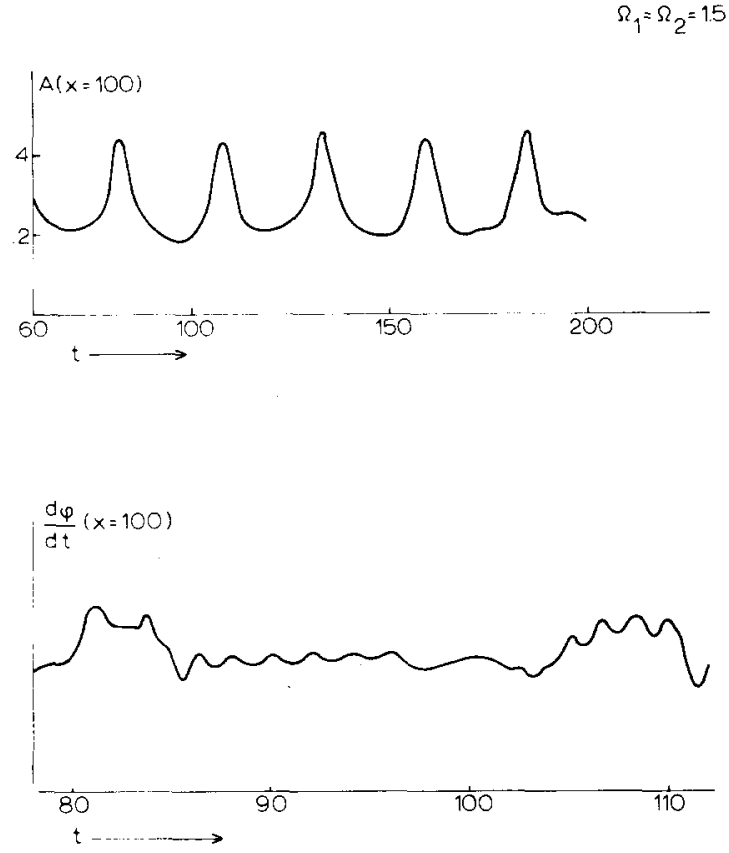

Fig. 2. The midpoint of the ss-breather as a function of time for the symmetric soliton-soliton collision.

considerable (a factor of 60 ) almost the same fraction of energy and charge was transferred to the ss-breather. On the average it was 0.14 with a variation from 0.135 for massive solitons to 0.15 for very light solitons. To see how stable the found ss-breather is we studied its time dependence for 1000 time units by simply removing the outgoing solitons when they are well separated from the ss-breather. This was done at $t=55$. Within the accuracy of our calculation the period and amplitude of the pulsations is stable and no evidence of dissipation of the ss-breather was found.

A completely different situation occurs in the antisymmetric soliton-soliton collision, where the antisymmetry of the total wavefunction precludes the production of a non-zero amplitude at $x=100$. The two solitons repel each other and after some deformation and time delay they are reflected elastically. We have found that the picture described above is specific for the pure antisymmetric case. If the solitons have different $\Omega_{i}$ but still with the phase $\theta=\pi$ the process is qualitatively the same as in the symmetric case. The solitons pass each other inelastically with the production of a breather. 


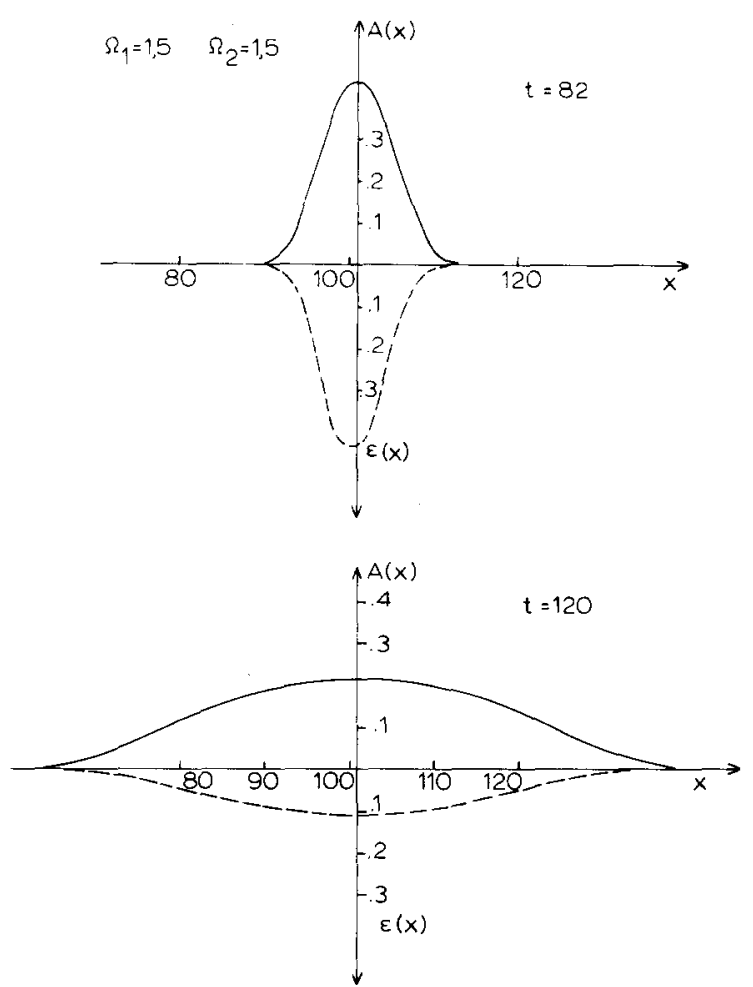

Fig. 3. Two cross sections of the symmetric ss-breather at the moments that it is largest and smallest in amplitude.

We now proceed to discuss the results for the case of the collision between a soliton and an anti-soliton. Again a pulsating object is produced (to be called a sabreather). The result is shown in fig. 4 in the $x-t$ plane. The energy of the sa-breather is around 0.14 of the total initial energy in the case that $\Omega_{1}=-\Omega_{2}=1.5$ while for the cases with different $\Omega_{i}$ the energy of the produced sa-breather can drop significantly when the $\Omega_{i}$ are different. For the case that the ratio of the soliton and antisoliton mass is 170 the collision process has a negligible inelasticity. The structure of the sabreather is quite different from that of the ss-breather. It has a much smaller charge $\left(Q_{\mathrm{m}} \approx 0\right.$ in the symmetric case). The ratio of the charge to the energy for the ssbreather is about 0.5 being close to that of the original solitons, whereas for the sa-breather this ratio is much smaller and depends on the parameter of the initial condition. Also the period of pulsation $\left(t_{\text {period }} \approx 2\right.$ in the symmetric case) is much smaller in this case.

In order to see whether these breathers are specific

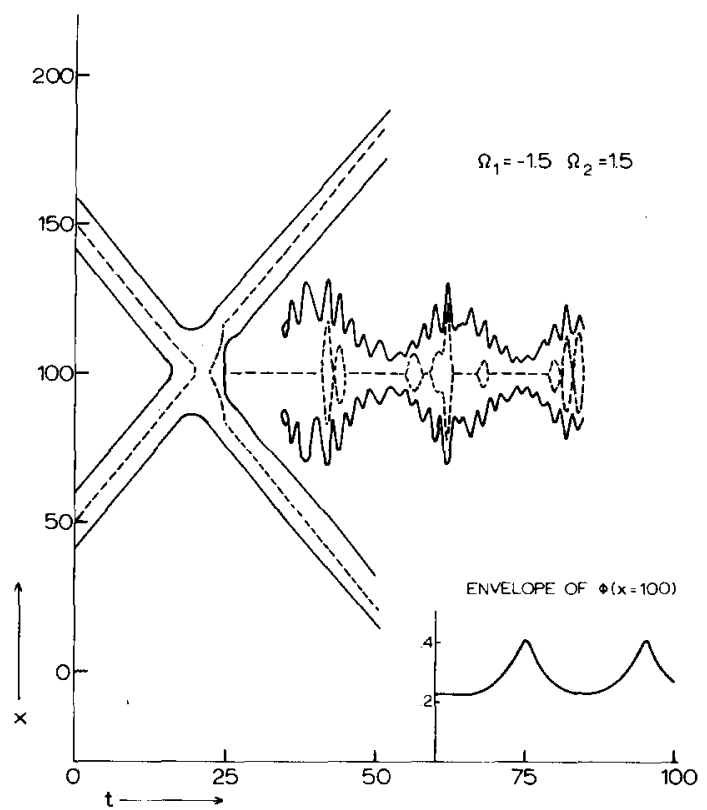

Fig. 4. The same as fig. 1, but for the symmetric soliton-antisoliton collision process. The subplot shows the change of the envelope of the sa-breather amplitude as a function of time.

to the model (5) chosen we have also studied the interaction (6). Similar results are obtained with the major difference that the inelasticity is much larger in this case. The fraction of energy transferred to the middle bump for $\nu^{2}=0.5$ was of the order of 0.9 , while for larger $\nu$ evidence is found that the two original solitons seem to fuse completely in the region of the collision.

In conclusion, we have seen that in the collision process of solitons other particle-like objects can be created, which seem to be stable or at least have an extremely long life time. To our knowledge this is the first time that the production of soliton-like objects in a classical relativistic field theoretical model has been observed. Although this has been found for one-dimen. sional models, we expect, since the solitons are also stable in higher dimensions, that we also should get similar phenomena there. For a non-relativistic model having gaussian-shape solitons the production of an additional soliton in the collision process of solitons has been reported on [7]. Also some evidence has been presented for the regularized long wave equation [8]. Some work has also recently been done in a chiral field theory [9] and in the three-wave problem [10] 
showing that a nontrivial interaction exists with the possibility of the decay of one soliton into more solitons.

Our results can be compared with the process of a kink-anti-kink collision, where objects with a long life time were found, called pulsons [11]. However, these objects are essentially unstable because a loss of energy was found for them. In our case, within the accuracy of our calculation, the energy of the breathers does not change even after $20-40$ oscillations. Another difference is that pulsons were found in kinkanti-kink collisions and not in kink-kink collisions. A more detailed version of this work will be presented elsewhere.

This work was done while one of the authors (Yu.S.) was on a two-month visit to the Netherlands in the framework of an agreement between the atomic commission of the USSR and the Reactor Centre in the Netherlands. This author would like to thank the Free University of Amsterdam for their warm hospitality.

\section{References}

[1] J. Werle, Phys. Lett. 71 B (1977) 357, 367.

[2] T.F. Morris, Phys. Lett. 76B (1978) 337; 78B (1978) 87.

[3] T.F. Morris, Can. J. Phys. 56 (1978) 1405.

[4] A. Chodos, R.L. Jaffe, K. Johnson, C.B. Thorn and V. Weisskopf, Phys. Rev. D9 (1974) 3471.

[5] R. Jackiw, Rev. Mod. Phys. 49 (1977) 681.

[6] R. Friedberg, T.D. Lee and A. Sirlin, Phys. Rev. D13 (1976) 2739.

[7] I. Bialynicki-Birula and J. Mycielsky, Ann. Phys. (NY) 100 (1976) 2;

J. Oficjalski and I. Bialynicki-Birula, Acta Phys. Pol. B9 (1978) 759.

[8] A.R. Santarelli, Nuovo Cimento 46B (1978) 179.

[9] V.E. Zakharov and A.V. Mikhailov, JETP Lett. 23 (1978) 47.

[10] V.E. Zakharov and S.V. Manakov, Sov. Phys. JETP 42 (1975) 842.

[11] For a review and references see: V.G. Makhankov, Phys. Rep. 35C (1978) 1. 